\title{
Gas-Phase Synthesis of Pyrazolo[3,4-b]pyridin-4-ones
}

\author{
Martha Mackay \\ Andrew Nortcliffe \\ Hamish McNab' \\ Alison N. Hulme*
}

EastCHEM School of Chemistry, The University of Edinburgh,

West Mains Road, Edinburgh EH9 3J, UK

Alison.Hulme@ed.ac.uk

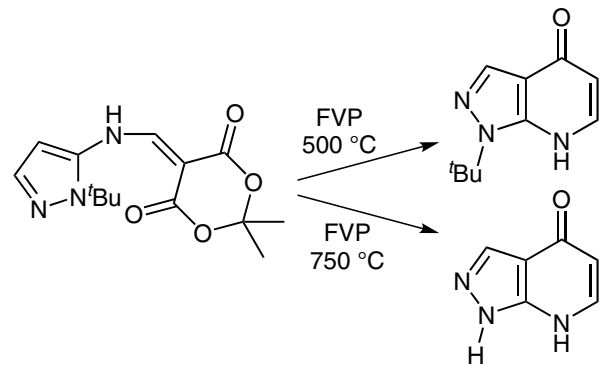

trogen atom to provide a useful route to the pyrazolo[1,5a]pyrimidine system 5 (Scheme 1 ). ${ }^{5}$ Clearly this route must be blocked to provide pyrazolo[3,4-b]pyridin-4-ones.

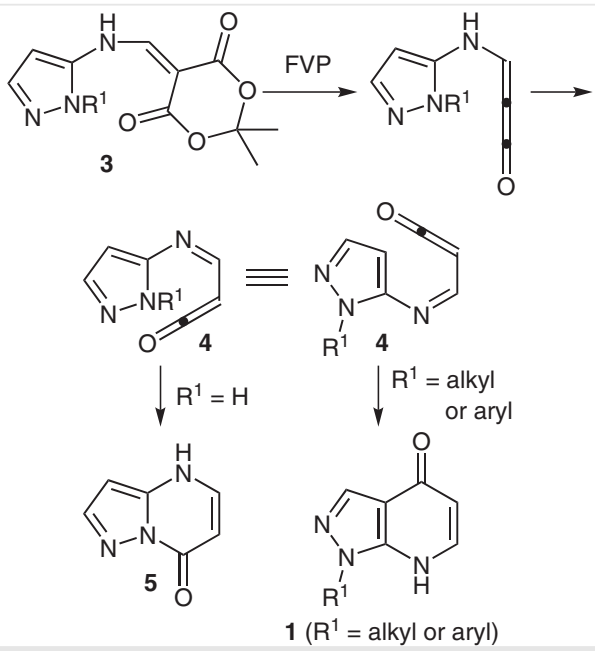

Scheme 1

The present work therefore had a range of objectives. First, $3\left(\mathrm{R}^{1}=\right.$ alkyl or aryl $)$ were synthesized and pyrolyzed to ensure that, in the absence of the pyrazole $\mathrm{NH}$, cyclization onto the adjacent carbon atom to give $\mathbf{1}(\mathrm{R}=1$-alkyl or 1 -aryl) would take place (Scheme 1), as observed in many related systems. ${ }^{6}$ Second, we explored the design of a thermal N-protecting group, which would remain at low furnace temperatures, but be selectively removed at higher furnace temperatures to provide $\mathrm{N}$-unsubstituted pyrazolopyridinones $\mathbf{1}\left(\mathrm{R}^{1}=\mathrm{H}\right)$. If the previous stages were successful, we aimed finally to functionalize the 4-position of the pyrazolo[3,4-b]pyridin-4-ones to establish that the route has significant potential for the synthesis of pyrazolo[3,4$b]$ pyridines 2 . 
The 1-substituted and 1,3-disubstituted 3-aminopyrazoles 6a-f (Figure 2) were either commercially available or were synthesized by known methods. Compounds $\mathbf{6 c},{ }^{7} \mathbf{6 e},{ }^{8}$ and $\mathbf{6}^{9 \mathrm{a}}$ are known only in patents or are formed in poor yield; ${ }^{9 b}$ their full characterization data are given here. Compound $\mathbf{6 c}$ was formed as a 5:1 mixture of $\mathbf{6 c}$ and its 1-tertbutyl-3-amino isomer, which was taken on to the next stage without purification. Reaction of $\mathbf{6 a - f}$ with methoxymethylene Meldrum's acid in acetonitrile gave the aminomethylene derivatives 3a-f (Figure 2) in $89-99 \%$ yield and (generally) high purity. Compound $\mathbf{3 c}$ was purified by recrystallization before pyrolysis.
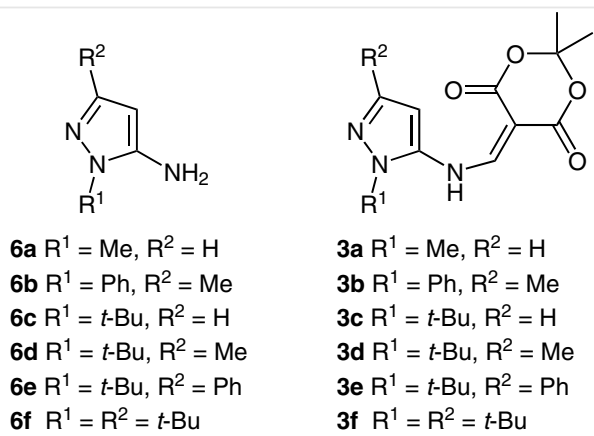

3a $R^{1}=M e, R^{2}=H$

$3 b R^{1}=P h, R^{2}=M e$

$3 c \mathrm{R}^{1}=t-\mathrm{Bu}, \mathrm{R}^{2}=\mathrm{H}$

3d $\mathrm{R}^{1}=t-\mathrm{Bu}, \mathrm{R}^{2}=\mathrm{Me}$

3e $R^{1}=t-B u, R^{2}=P h$

3f $\mathrm{R}^{1}=\mathrm{R}^{2}=t$-Bu

Figure 2 1,3-Disubstituted 3-aminopyrazoles 6 and aminomethylene derivatives 3

FVP of $3 \mathbf{a}$ and $\mathbf{3 b}$ at $600{ }^{\circ} \mathrm{C}(0.03$ Torr $)$ gave 1-methylpyrazolo[3,4-b]pyridin-4-one (1aa) (92\%) and its 3-methyl-1phenyl analogue $\mathbf{1 b a}$ (95\%), respectively, as involatile solids that crystallized at the exit point of the furnace. It is clear, therefore, that blocking the 1-position of the pyrazole has the effect of diverting the cyclization to the adjacent carbon atom to provide the target pyrazolopyridinones.

In order to access the 1-unsubstituted pyrazolo[3,4b]pyridines $\mathbf{1}\left(\mathrm{R}^{1}=\mathrm{H}\right)$, a thermally removable $\mathrm{N}$-protecting group was required. If a retro-ene reaction is possible, an $\mathrm{N}$ tert-butyl group is ideal because the only co-product is 2methyl-1-propene. We have exploited this in the pyridazin3 -one series ${ }^{10}$ and it is also known that $\mathrm{N}$-tert-butylpyrazole (7) loses 2-methyl-1-propene at high temperatures (Scheme 2). ${ }^{11} \mathrm{~A}$ temperature profile of this reaction (Figure 3 ) shows that, in our apparatus, at temperatures below $600{ }^{\circ} \mathrm{C}$ the $\mathrm{N}$-alkyl product 7 is formed exclusively whereas at temperatures above $850^{\circ} \mathrm{C}$, only the deprotected product 8 was formed. It was therefore anticipated that FVP of $\mathbf{3 c - f}$ in the range $500-600{ }^{\circ} \mathrm{C}$ should provide the $N$-tert-butyl products $1\left(\mathrm{R}^{1}=t-\mathrm{Bu}\right)$ whereas FVP in the range $750-850{ }^{\circ} \mathrm{C}$ should provide the deprotected products $\mathbf{1}\left(R^{1}=H\right)$.

These predictions were borne out in practice. FVP of $\mathbf{3 c}-$ f at $500{ }^{\circ} \mathrm{C}$ gave the pyrazolopyridinones $1 \mathbf{c a}, 1 \mathbf{d a}, 1 \mathbf{1 e a}$, and 1fa (Figure 4) in $83-97 \%$ yields and at $750-850{ }^{\circ} \mathrm{C}$ gave the deprotected products $\mathbf{1 c b}$ and $\mathbf{1 d b}$ in $67-82 \%$ yields whilst the more highly substituted derivatives $\mathbf{1 e b}$ and $\mathbf{1 f b}$ were

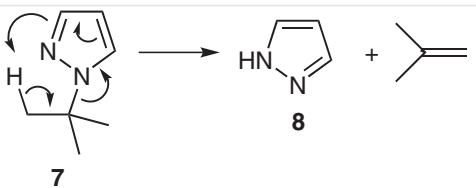

Scheme 2

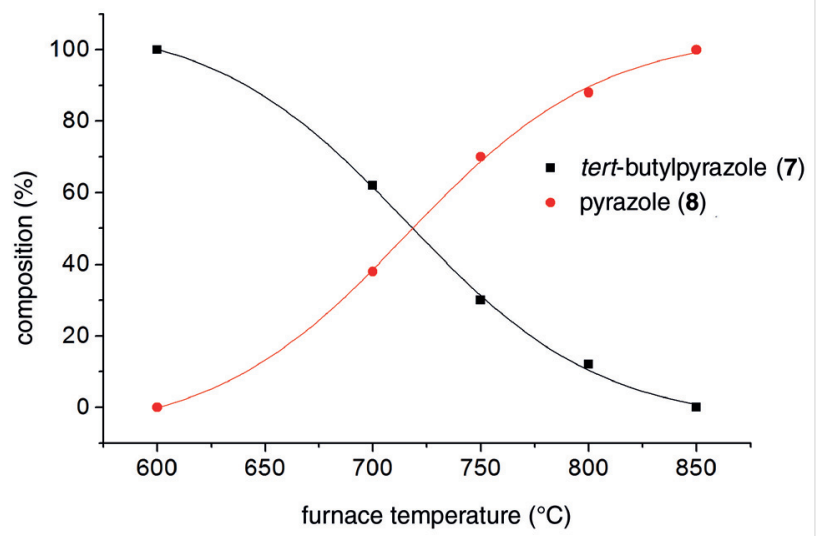

Figure 3 Temperature-conversion plot for FVP of $\mathbf{7}$

obtained as more complex mixtures. ${ }^{12} \mathrm{~N}$-Unsubstituted pyrazolopyridinones show exceptionally broad peaks in their NMR spectra due to tautomerization, but the two $\mathrm{NH}$ resonances at ca. $\delta_{\mathrm{H}}=12.8-13.8$ and $11.5-11.8$ are characteristic, as previously reported. ${ }^{2 a}$<smiles>[R]c1nn([Tl])c2[nH]ccc(=O)c12</smiles>

$$
\begin{array}{lll}
\text { 1aa } R^{1}=M e, R^{2}=H & & \\
{\text { 1ba } R^{1}=P h, R^{2}=M e} & & \\
\text { 1ca } R^{1}=t-B u, R^{2}=H & & \text { 1cb }^{1}=H, R^{2}=H \\
\text { 1da } R^{1}=t-B u, R^{2}=M e & & \text { 1db } R^{1}=H, R^{2}=M e \\
\text { 1ea } R^{1}=t-B u, R^{2}=P h & & \text { 1eb } R^{1}=H, R^{2}=P h \\
\text { 1fa } R^{1}=R^{2}=t-B u & & \text { 1fb } R^{1}=H, R^{2}=t-B u
\end{array}
$$

Figure 4 Pyrazolopyridinones 1

As an alternative to the one-pass cyclization-deprotection described above, the protecting group can be retained prior to functionalization of the 4-oxo substituent. This strategy is illustrated for the 3-phenyl series $\mathbf{1 e}$, which was chosen as it might prove unreactive owing to peri interactions with the 3-substituent.

Thus, treatment of 1ea with phosphoryl chloride gave the 4-chloro compound $\mathbf{9}(99 \%)$, which could either be thermally deprotected to $\mathbf{1 0}(87 \%)$, or reacted further. For example, reaction of $\mathbf{9}$ with pyrrolidine in the absence of a catalyst provided a low yield of the pyrrolidino compound $\mathbf{1 1}$ (37\%); alternatively, reaction with aniline under Buchwald- 
Hartwig conditions gave the anilino compound 12 (87\%), which could be thermally deprotected to $\mathbf{1 3}$ (72\%) (Scheme 3).

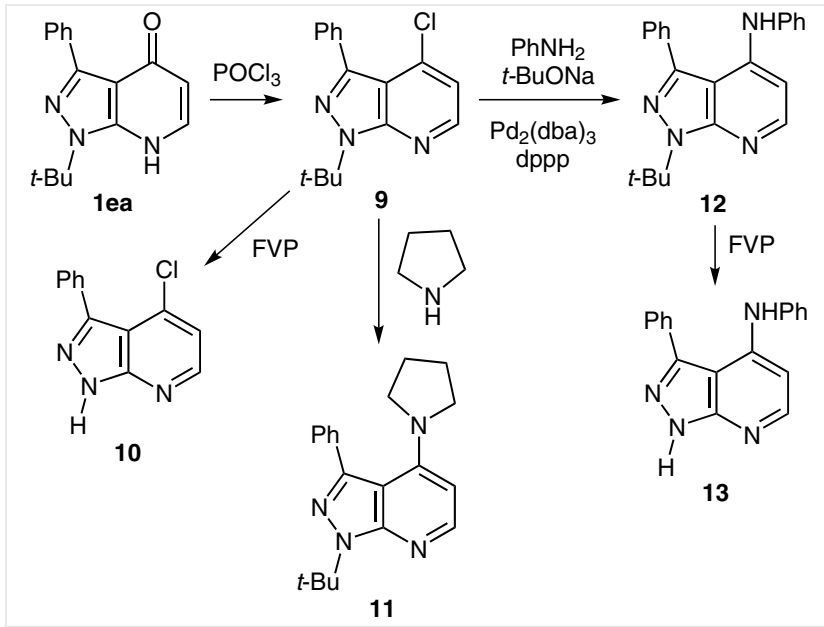

Scheme 3

In conclusion, the work described here has provided a flexible gas-phase route to pyrazolo[3,4- $b]$ pyridin-4-ones and pyrazolo[3,4-b]pyridines. An important feature of the strategy is the use of an $N$-tert-butyl group, which may be retained at low furnace temperatures (allowing functionalization of the 4-oxo group) or removed at high furnace temperatures to provide a one-pass route to $\mathrm{N}$-unsubstituted analogues.

${ }^{1} \mathrm{H}$ and ${ }^{13} \mathrm{C}$ NMR spectra were recorded at 500 or $250 \mathrm{MHz}$ and 125 or $63 \mathrm{MHz}$, respectively, unless otherwise stated. Chemical shifts are given in ppm relative to TMS. Mass spectra were recorded under electron impact conditions.

\section{5-Amino-1-tert-butyl-1H-pyrazole (6c) ${ }^{7}$}

tert-Butylhydrazine hydrochloride (5.99 g, $48.1 \mathrm{mmol}$ ) was added to EtOH $(60 \mathrm{~mL})$ to form a slurry. To this was added $\mathrm{NaOAc}(7.93 \mathrm{~g}, 96.7$ $\mathrm{mmol}$ ) and 2-chloroacrylonitrile ( $5 \mathrm{~mL}, 62.6 \mathrm{mmol}$ ). The solution was heated to $80{ }^{\circ} \mathrm{C}$ for $18 \mathrm{~h}$, cooled, and the solvent removed in vacuo. The residue was slowly diluted with distilled $\mathrm{H}_{2} \mathrm{O}(35 \mathrm{~mL})$ and partitioned between sat. aq $\mathrm{NaHCO}_{3}(40 \mathrm{~mL})$ and EtOAc $(40 \mathrm{~mL})$. The organic layer was separated and the aqueous layer was extracted with EtOAc $(2 \times 20 \mathrm{~mL})$. The organic layers were combined, washed with brine $(20 \mathrm{~mL})$, dried $\left(\mathrm{MgSO}_{4}\right)$, and the solvent removed in vacuo to afford a red oil; yield: $7.95 \mathrm{~g}(91 \%)$; bp $93-94{ }^{\circ} \mathrm{C} / 0.9$ Torr (yellow liquid). The product was a 5:1 mixture of the title compound $\mathbf{6 c}$ and its 1 tert-butyl-3-amino isomer. The crude product was used to prepare the Meldrum's acid derivative $\mathbf{3 c}$, which was purified by recrystallization (see below).

${ }^{1} \mathrm{H} \mathrm{NMR}\left(\mathrm{CDCl}_{3}\right): \delta=7.21\left(\mathrm{~d},{ }^{3} \mathrm{~J}=1.8 \mathrm{~Hz}, 1 \mathrm{H}\right), 5.57\left(\mathrm{~d},{ }^{3} \mathrm{~J}=1.8 \mathrm{~Hz}, 1 \mathrm{H}\right)$, 3.60 (br s, $2 \mathrm{H}$ ), 1.65 (s, $9 \mathrm{H}$ ).

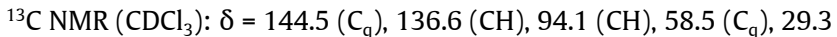
(3 $\mathrm{CH}_{3}$ ).

MS: $m / z(\%)=139\left(\mathrm{M}^{+}, 45\right), 83\left(\mathrm{M}-\mathrm{C}_{4} \mathrm{H}_{10}, 100\right)$.
HRMS: $m / z$ calcd for $\mathrm{C}_{7} \mathrm{H}_{13} \mathrm{~N}_{3}\left(\mathrm{M}^{+}\right)$: 139.1104 ; found: 139.1103 .

\section{5-Amino-1-tert-butyl-3-phenyl-1H-pyrazole $(6 e)^{8}$}

A solution of 3-oxo-3-phenylpropanenitrile $(1.5 \mathrm{~g}, 10.3 \mathrm{mmol})$ in EtOH $(10 \mathrm{~mL})$ was added to a slurry of tert-butylhydrazine hydrochloride $(2.6 \mathrm{~g}, 20.7 \mathrm{mmol})$ in $\mathrm{EtOH}(35 \mathrm{~mL})$ and the solution was heated to reflux with stirring for $18 \mathrm{~h}$. The solution was cooled, concentrated and the residue was partitioned between sat. aq $\mathrm{NaHCO}_{3}(30 \mathrm{~mL})$ and EtOAc ( $30 \mathrm{~mL})$. The organic layer was separated and the aqueous layer was extracted with EtOAc $(2 \times 20 \mathrm{~mL})$. The organic layers were combined, washed with brine $(20 \mathrm{~mL})$, dried $\left(\mathrm{MgSO}_{4}\right)$, and the solvent removed in vacuo to give $\mathbf{6 e}$ as a pale yellow solid; yield: $2.2 \mathrm{~g}(97 \%)$; mp $100-102{ }^{\circ} \mathrm{C}$.

${ }^{1} \mathrm{H}$ NMR (DMSO- $\left.d_{6}\right): \delta=7.64\left(\mathrm{~d},{ }^{3} J=7.4 \mathrm{~Hz}, 2 \mathrm{H}\right), 7.33\left(\mathrm{t},{ }^{3} J=7.4 \mathrm{~Hz}, 2\right.$ H), $7.22\left(\mathrm{t},{ }^{3} J=7.4 \mathrm{~Hz}, 1 \mathrm{H}\right), 5.79(\mathrm{~s}, 1 \mathrm{H}), 4.97(\mathrm{~s}, 2 \mathrm{H}), 1.58(\mathrm{~s}, 9 \mathrm{H})$.

${ }^{13} \mathrm{C}$ NMR (DMSO- $\left.d_{6}\right): \delta=148.1\left(\mathrm{C}_{\mathrm{q}}\right), 146.1\left(\mathrm{C}_{\mathrm{q}}\right), 135.0\left(\mathrm{C}_{\mathrm{q}}\right), 128.8(2$ $\mathrm{CH}), 127.1(\mathrm{CH}), 125.0(2 \mathrm{CH}) 89.2(\mathrm{CH}), 58.3\left(\mathrm{C}_{\mathrm{q}}\right), 40.1\left(3 \mathrm{CH}_{3}\right)$.

MS: $m / z(\%)=215\left(\mathrm{M}^{+}, 25\right), 159(100)$.

HRMS: $m / z$ calcd for $\mathrm{C}_{13} \mathrm{H}_{17} \mathrm{~N}_{3}\left(\mathrm{M}^{+}\right)$: 215.1417; found: 215.1416 .

\section{5-Amino-1,3-di-tert-butyl-1H-pyrazole $(6 \mathrm{f})^{9}$}

A solution of 4,4-dimethyl-3-oxovaleronitrile $(1.75 \mathrm{~g}, 14.0 \mathrm{mmol})$ in EtOH $(10 \mathrm{~mL})$ was added to a slurry of tert-butylhydrazine hydrochloride ( 3.5 g. $28.1 \mathrm{mmol})$ in $\mathrm{EtOH}(35 \mathrm{~mL})$ and the solution was heated to reflux with stirring for $18 \mathrm{~h}$. The solution was cooled, concentrated, and the residue was partitioned between sat. aq $\mathrm{NaHCO}_{3}(30 \mathrm{~mL})$ and EtOAc $(30 \mathrm{~mL})$. The organic layer was separated and the aqueous layer was extracted with EtOAc $(2 \times 20 \mathrm{~mL})$. The organic layers were combined, washed with brine $(20 \mathrm{~mL})$, dried $\left(\mathrm{MgSO}_{4}\right)$, and the solvent removed to give $\mathbf{6 f}$ as a pale orange solid; yield: $1.8 \mathrm{~g}$ (66\%); $\mathrm{mp} 67-$ $69{ }^{\circ} \mathrm{C}$ ( Lit. ${ }^{9 a} \mathrm{mp} 64-66{ }^{\circ} \mathrm{C}$ ).

${ }^{1} \mathrm{H} \mathrm{NMR}\left(\mathrm{CDCl}_{3}\right): \delta=5.48(\mathrm{~s}, 1 \mathrm{H}), 3.46(\mathrm{~s}, 2 \mathrm{H}), 1.27(\mathrm{~s}, 18 \mathrm{H})$.

${ }^{13} \mathrm{C} \mathrm{NMR}\left(\mathrm{CDCl}_{3}\right): \delta=157.8\left(\mathrm{C}_{\mathrm{q}}\right), 144.1\left(\mathrm{C}_{\mathrm{q}}\right), 90.2(\mathrm{CH}), 58.2\left(\mathrm{C}_{\mathrm{q}}\right), 44.7$ $\left(\mathrm{C}_{\mathrm{q}}\right), 30.4\left(3 \mathrm{CH}_{3}\right), 29.5\left(3 \mathrm{CH}_{3}\right)$.

Spectra differ significantly from those reported, ${ }^{9 b}$ but were recorded in a different solvent.

MS: $m / z(\%)=195\left(\mathrm{M}^{+}, 29\right), 139$ (63), 124 (100).

HRMS: $m / z$ calcd for $\mathrm{C}_{11} \mathrm{H}_{21} \mathrm{~N}_{3}\left(\mathrm{M}^{+}\right)$: 195.1730 ; found: 175.1728 .

\section{Meldrum's Acid Derivatives; General Procedure}

5-(Methoxymethylene)-2,2-dimethyl-1,3-dioxane-4,6-dione (0.5 g, $2.9 \mathrm{mmol}$ ) was added to a stirred solution of the 5-aminopyrazole 6 $(2.9 \mathrm{mmol})$ in $\mathrm{MeCN}(10 \mathrm{~mL})$. After stirring for $1 \mathrm{~h}$, the solvent was removed in vacuo to complete the precipitation of the product.

5-(1-Methyl-1H-pyrazol-5-ylaminomethylene)-2,2-dimethyl-1,3dioxane-4,6-dione (3a)

Treatment of $\mathbf{6 a}$ using the general procedure gave $3 \mathbf{a}$; yield: $0.71 \mathrm{~g}$ (98\%); yellow solid; mp $144{ }^{\circ} \mathrm{C}(\mathrm{MeOH})$.

${ }^{1} \mathrm{H} \mathrm{NMR}\left(\mathrm{CDCl}_{3}\right): \delta=11.28\left(\mathrm{~d},{ }^{3} \mathrm{~J}=13.3 \mathrm{~Hz}, 1 \mathrm{H}\right), 8.34\left(\mathrm{~d},{ }^{3} \mathrm{~J}=13.3 \mathrm{~Hz}, 1\right.$ $\mathrm{H}), 7.34\left(\mathrm{~d},{ }^{3} \mathrm{~J}=2.1 \mathrm{~Hz}, 1 \mathrm{H}\right), 6.18\left(\mathrm{~d},{ }^{3} \mathrm{~J}=2.1 \mathrm{~Hz}, 1 \mathrm{H}\right), 3.85(\mathrm{~s}, 3 \mathrm{H}), 1.75$ $(\mathrm{s}, 6 \mathrm{H})$.

${ }^{13} \mathrm{C}$ NMR $\left(\mathrm{CDCl}_{3}\right): \delta=165.7\left(\mathrm{C}_{\mathrm{q}}\right), 162.6\left(\mathrm{C}_{\mathrm{q}}\right), 154.7(\mathrm{CH}), 139.1(\mathrm{CH})$, $138.0\left(\mathrm{C}_{\mathrm{q}}\right), 105.6\left(\mathrm{C}_{\mathrm{q}}\right), 95.3(\mathrm{CH}), 88.9\left(\mathrm{C}_{\mathrm{q}}\right), 35.4\left(\mathrm{CH}_{3}\right), 27.1\left(2 \mathrm{CH}_{3}\right)$.

MS: $m / z(\%)=251\left(\mathrm{M}^{+}, 16\right), 193(100), 149(14), 122(40)$.

Anal. Calcd for $\mathrm{C}_{11} \mathrm{H}_{13} \mathrm{~N}_{3} \mathrm{O}_{4}$ : C, 52.6; $\mathrm{H}, 5.2 ; \mathrm{N}, 16.75$. Found: $\mathrm{C}, 52.65$; H, 5.35; N, 16.8 . 
5-(3-Methyl-1-phenyl-1H-pyrazol-5-ylaminomethylene)-2,2-dimethyl-1,3-dioxane-4,6-dione (3b)

Treatment of $\mathbf{6 b}$ using the general procedure gave $\mathbf{3 b}$; yield: $0.90 \mathrm{~g}$ (95\%); yellow solid; $\mathrm{mp} 167^{\circ} \mathrm{C}(\mathrm{MeOH})$.

${ }^{1} \mathrm{H} \mathrm{NMR}\left(\mathrm{CDCl}_{3}\right): \delta=11.45\left(\mathrm{~d},{ }^{3} \mathrm{~J}=13.4 \mathrm{~Hz}, 1 \mathrm{H}\right), 8.38\left(\mathrm{~d},{ }^{3} \mathrm{~J}=13.4 \mathrm{~Hz}, 1\right.$ H), 7.56-7.42 (m, 5 H), 6.17 (s, $1 \mathrm{H}), 2.34(\mathrm{~s}, 3 \mathrm{H}), 1.71(\mathrm{~s}, 6 \mathrm{H})$.

${ }^{13} \mathrm{C}$ NMR $\left(\mathrm{CDCl}_{3}\right): \delta=165.3\left(\mathrm{C}_{\mathrm{q}}\right), 162.7\left(\mathrm{C}_{\mathrm{q}}\right), 153.1(\mathrm{CH}), 150.1\left(\mathrm{C}_{\mathrm{q}}\right)$, $138.3\left(\mathrm{C}_{\mathrm{q}}\right), 136.8\left(\mathrm{C}_{\mathrm{q}}\right), 130.0(2 \mathrm{CH}), 128.8(\mathrm{CH}), 124.8(2 \mathrm{CH}), 105.5$ $\left(\mathrm{C}_{\mathrm{q}}\right), 94.3(\mathrm{CH}), 88.8\left(\mathrm{C}_{\mathrm{q}}\right), 27.1\left(2 \mathrm{CH}_{3}\right), 14.0\left(\mathrm{CH}_{3}\right)$.

MS: $m / z(\%)=327\left(\mathrm{M}^{+}, 23\right), 269$ (100), 225 (22), 184 (74), 156 (22).

Anal. Calcd for $\mathrm{C}_{17} \mathrm{H}_{17} \mathrm{~N}_{3} \mathrm{O}_{4}$ : C, 62.4; $\mathrm{H}, 5.25 ; \mathrm{N}, 12.85$. Found: $\mathrm{C}, 62.3$; H, 5.15; N, 12.75 .

\section{5-(1-tert-Butyl-1H-pyrazol-5-ylamino)methylene-2,2-dimethyl- 1,3-dioxane-5,6-dione (3c)}

Treatment of $\mathbf{6 c}$ using the general procedure gave $\mathbf{3 c}$; yield: $0.985 \mathrm{~g}$ (97\%); yellow solid; $\mathrm{mp} 84^{\circ} \mathrm{C}$.

${ }^{1} \mathrm{H}$ NMR $\left(\mathrm{CDCl}_{3}\right): \delta=11.53\left(\mathrm{~d},{ }^{3} \mathrm{~J}=13.4 \mathrm{~Hz}, 1 \mathrm{H}\right), 8.33\left(\mathrm{~d},{ }^{3} \mathrm{~J}=13.4 \mathrm{~Hz}, 1\right.$ H), $7.41\left(\mathrm{~d},{ }^{3} \mathrm{~J}=1.9 \mathrm{~Hz}, 1 \mathrm{H}\right), 6.23\left(\mathrm{~d},{ }^{3} \mathrm{~J}=1.9 \mathrm{~Hz}, 1 \mathrm{H}\right), 1.77(\mathrm{~s}, 6 \mathrm{H}), 1.70$ (s, $9 \mathrm{H})$.

${ }^{13} \mathrm{C}$ NMR $\left(\mathrm{CDCl}_{3}\right): \delta=165.6\left(\mathrm{C}_{\mathrm{q}}\right), 162.9\left(\mathrm{C}_{\mathrm{q}}\right), 154.7(\mathrm{CH}), 137.5\left(\mathrm{C}_{\mathrm{q}}\right)$, $137.3(\mathrm{CH}), 105.6\left(\mathrm{C}_{\mathrm{q}}\right), 97.8(\mathrm{CH}), 88.4\left(\mathrm{C}_{\mathrm{q}}\right), 60.3\left(\mathrm{C}_{\mathrm{q}}\right), 29.8\left(3 \mathrm{CH}_{3}\right), 27.3$ $\left(2 \mathrm{CH}_{3}\right)$.

MS: $m / z(\%)=293\left(\mathrm{M}^{+}, 21\right), 235$ (47), 179 (59), 161 (100).

HRMS: $m / z$ calcd for $\mathrm{C}_{14} \mathrm{H}_{19} \mathrm{~N}_{3} \mathrm{O}_{4}\left(\mathrm{M}^{+}\right)$: 293.1381; found: 293.1384 .

\section{5-(1-tert-Butyl-3-methyl-1H-pyrazol-5-ylamine)-2,2-dimethyl-} 1,3-dioxane-4,6-dione (3d)

Treatment of $\mathbf{6 d}$ using the general procedure gave 3d; yield: $0.88 \mathrm{~g}$ (99\%); yellow solid; $\mathrm{mp} 82^{\circ} \mathrm{C}$.

${ }^{1} \mathrm{H} \mathrm{NMR}\left(\mathrm{CDCl}_{3}\right): \delta=11.47\left(\mathrm{br} \mathrm{d},{ }^{3} \mathrm{~J}=13.4 \mathrm{~Hz}, 1 \mathrm{H}\right), 8.29\left(\mathrm{~d},{ }^{3} \mathrm{~J}=13.4 \mathrm{~Hz}\right.$, $1 \mathrm{H}), 6.00(\mathrm{~s}, 1 \mathrm{H}), 2.23(\mathrm{~s}, 3 \mathrm{H}), 1.75(\mathrm{~s}, 6 \mathrm{H}), 1.66(\mathrm{~s}, 9 \mathrm{H})$.

${ }^{13} \mathrm{C}$ NMR $\left(\mathrm{CDCl}_{3}\right): \delta=165.9\left(\mathrm{C}_{\mathrm{q}}\right), 162.9\left(\mathrm{C}_{\mathrm{q}}\right), 154.4(\mathrm{CH}), 146.2\left(\mathrm{C}_{\mathrm{q}}\right)$, $137.7\left(\mathrm{C}_{\mathrm{q}}\right), 105.5\left(\mathrm{C}_{\mathrm{q}}\right), 97.1(\mathrm{CH}), 88.1\left(\mathrm{C}_{\mathrm{q}}\right), 59.7\left(\mathrm{C}_{\mathrm{q}}\right), 29.8\left(3 \mathrm{CH}_{3}\right), 27.1$ $\left(2 \mathrm{CH}_{3}\right), 13.9\left(\mathrm{CH}_{3}\right)$.

MS: $m / z(\%)=307\left(\mathrm{M}^{+}, 21\right), 249(100), 175(68)$

HRMS: $m / z$ calcd for $\mathrm{C}_{15} \mathrm{H}_{21} \mathrm{~N}_{3} \mathrm{O}_{4}\left(\mathrm{M}^{+}\right)$: 307.1527; found: 307.1533 .

\section{5-(1-tert-Butyl-3-phenyl-1H-pyrazol-5-ylamino)methylene-2,2-} dimethyl-1,3-dioxane-4,6-dione (3e)

Treatment of $\mathbf{6 e}$ using the general procedure gave $\mathbf{3 e}$; yield: $0.97 \mathrm{~g}$ (98\%); yellow solid; mp $152{ }^{\circ} \mathrm{C}$.

${ }^{1} \mathrm{H}$ NMR $\left(\mathrm{CDCl}_{3}\right): \delta=11.59\left(\mathrm{~d},{ }^{3} \mathrm{~J}=13.4 \mathrm{~Hz}, 1 \mathrm{H}\right), 8.43\left(\mathrm{~d},{ }^{3} \mathrm{~J}=13.4 \mathrm{~Hz}, 1\right.$ $\mathrm{H}), 7.80\left(\mathrm{~d},{ }^{3} \mathrm{~J}=7.3 \mathrm{~Hz}, 2 \mathrm{H}\right), 7.44\left(\mathrm{t},{ }^{3} \mathrm{~J}=7.3 \mathrm{~Hz}, 2 \mathrm{H}\right), 7.35\left(\mathrm{t},{ }^{3} \mathrm{~J}=7.3 \mathrm{~Hz}\right.$, $1 \mathrm{H}), 6.75(\mathrm{~s}, 1 \mathrm{H}), 1.81(\mathrm{~s}, 6 \mathrm{H}), 1.77(\mathrm{~s}, 9 \mathrm{H})$.

${ }^{13} \mathrm{C} \mathrm{NMR}\left(\mathrm{CDCl}_{3}\right): \delta=166.0\left(\mathrm{C}_{\mathrm{q}}\right), 162.9\left(\mathrm{C}_{\mathrm{q}}\right), 154.4(\mathrm{CH}), 148.5\left(\mathrm{C}_{\mathrm{q}}\right)$, $138.8\left(\mathrm{C}_{\mathrm{q}}\right), 132.8\left(\mathrm{C}_{\mathrm{q}}\right), 128.7(2 \mathrm{CH}), 128.1(\mathrm{CH}), 125.3(2 \mathrm{CH}), 105.7$ $\left(\mathrm{C}_{\mathrm{q}}\right), 94.5(\mathrm{CH}), 88.5\left(\mathrm{C}_{\mathrm{q}}\right), 60.6\left(\mathrm{C}_{\mathrm{q}}\right), 29.9\left(3 \mathrm{CH}_{3}\right), 27.2\left(2 \mathrm{CH}_{3}\right)$.

MS: $m / z(\%)=369\left(\mathrm{M}^{+}, 33\right), 311$ (100), 237 (58), 211 (57), 183 (44), 108 (45).

HRMS: $m / z$ calcd for $\mathrm{C}_{20} \mathrm{H}_{23} \mathrm{~N}_{3} \mathrm{O}_{4}\left(\mathrm{M}^{+}\right)$: 369.1683; found: 369.1690 .

5-(1,3-Di-tert-butyl-1H-pyrazol-5-ylamino)methylene-2,2-dimethyl-1,3-dioxane-5,6-dione (3f)

Treatment of $\mathbf{6 f}$ using the general procedure gave 3f, yield: $0.83 \mathrm{~g}$ (89\%); yellow solid; mp $105^{\circ} \mathrm{C}$.
${ }^{1} \mathrm{H} \mathrm{NMR}\left(\mathrm{CDCl}_{3}\right): \delta=11.47\left(\mathrm{~d},{ }^{3} \mathrm{~J}=13.1 \mathrm{~Hz}, 1 \mathrm{H}\right), 8.35\left(\mathrm{~d},{ }^{3} \mathrm{~J}=13.1 \mathrm{~Hz}, 1\right.$ H), $6.08(\mathrm{~s}, 1 \mathrm{H}), 1.78(\mathrm{~s}, 6 \mathrm{H}), 1.67(\mathrm{~s}, 9 \mathrm{H}), 1.28(\mathrm{~s}, 9 \mathrm{H})$.

${ }^{13} \mathrm{C}$ NMR $\left(\mathrm{CDCl}_{3}\right): \delta=165.9\left(\mathrm{C}_{\mathrm{q}}\right), 163.1\left(\mathrm{C}_{\mathrm{q}}\right), 158.9\left(\mathrm{C}_{\mathrm{q}}\right), 154.6(\mathrm{CH})$, $137.0\left(\mathrm{C}_{\mathrm{q}}\right), 105.5\left(\mathrm{C}_{\mathrm{q}}\right), 94.0(\mathrm{CH}), 87.8\left(\mathrm{C}_{\mathrm{q}}\right), 59.8\left(\mathrm{C}_{\mathrm{q}}\right), 32.3\left(\mathrm{C}_{\mathrm{q}}\right), 30.3(3$ $\left.\mathrm{CH}_{3}\right), 29.9\left(3 \mathrm{CH}_{3}\right), 27.1\left(2 \mathrm{CH}_{3}\right)$.

MS: $m / z(\%)=349\left(\mathrm{M}^{+}, 24\right), 291$ (100), 217 (27), 202 (45), $176(56)$.

HRMS: $m / z$ calcd for $\mathrm{C}_{18} \mathrm{H}_{27} \mathrm{~N}_{3} \mathrm{O}_{4}\left(\mathrm{M}^{+}\right)$: 349.1996; found: 349.2000 .

\section{FVP Reactions}

Flash vacuum pyrolysis reactions were carried out by distillation of the substrate in vacuo through an electrically heated silica furnace tube $(35 \times 2.5 \mathrm{~cm})$. Products were trapped in a U-tube situated at the exit point of the furnace and cooled with liquid $\mathrm{N}_{2}$. Conditions were first established on a small scale $(20 \mathrm{mg})$ where the product(s) were dissolved in a deuterated solvent and analyzed directly by ${ }^{1} \mathrm{H}$ NMR spectroscopy. Larger-scale pyrolyses, involving $0.1 \mathrm{~g}$ or more of substrate, were usually removed from the trap by solution in $\mathrm{CH}_{2} \mathrm{Cl}_{2}$ (30 $\mathrm{mL}$ ). The precursors and pyrolysis conditions [quantity of precursor, inlet temperature $\left(T_{\mathrm{i}}\right)$, furnace temperature $\left(T_{\mathrm{f}}\right)$, pressure range $(P)$, and pyrolysis time $(t)]$ and yields are stated.

\section{FVP of 1-tert-Butylpyrazole (7)}

This compound was too volatile for normal inlet conditions. It was therefore cooled in an acetone-dry ice bath, which was slowly removed to allow sublimation (20 mg, $T_{\mathrm{i}}$ acetone/dry ice bath, $T_{\mathrm{f}} 600-$ $850{ }^{\circ} \mathrm{C}, P 0.03$ Torr, $\left.t 15 \mathrm{~min}\right)$.

\section{1-Methyl-1,7-dihydropyrazolo[3,4-b]pyridin-4-one (1aa)}

FVP of 3a (recrystallized from $\mathrm{MeOH}, 203 \mathrm{mg}, 0.81 \mathrm{mmol}, T_{\mathrm{i}} 199{ }^{\circ} \mathrm{C}, T_{\mathrm{f}}$ $600{ }^{\circ} \mathrm{C}, P 0.03 \mathrm{Torr}, t 30 \mathrm{~min}$ ) gave 1aa; yield: $111 \mathrm{mg}(92 \%)$; off-white solid; mp $164{ }^{\circ} \mathrm{C}$ (Lit. $\left.{ }^{13} \mathrm{mp} 165-168{ }^{\circ} \mathrm{C}\right)$.

${ }^{1} \mathrm{H}$ NMR (DMSO- $\left.d_{6}\right): \delta=8.37\left(\mathrm{~d},{ }^{3} \mathrm{~J}=4.0 \mathrm{~Hz}, 1 \mathrm{H}\right.$ ), $8.30(\mathrm{~s}, 1 \mathrm{H}), 6.65(\mathrm{br}$ $\mathrm{s}, 1 \mathrm{H}), 4.22(\mathrm{~s}, 3 \mathrm{H})$

${ }^{13} \mathrm{C}$ NMR (DMSO- $\left.d_{6}\right): \delta=162.4\left(\right.$ br C $\left._{\mathrm{q}}\right), 150.7\left(\right.$ br C $\left._{\mathrm{q}}\right), 148.1(\mathrm{br} \mathrm{CH})$, 130.6 (CH), $107.9\left(\mathrm{br} \mathrm{C}_{\mathrm{q}}\right), 104.4(\mathrm{CH}), 33.9\left(\mathrm{CH}_{3}\right)$.

MS: $m / z(\%)=149\left(\mathrm{M}^{+}, 100\right), 95(12), 78(14), 63$ (13).

HRMS: $m / z$ calcd for $\mathrm{C}_{7} \mathrm{H}_{7} \mathrm{~N}_{3} \mathrm{O}\left(\mathrm{M}^{+}\right)$: 149.0584 ; found: 149.0584 .

\section{3-Methyl-1,7-dihydro-1-phenylpyrazolo[3,4-b]pyridin-4-one} (1ba)

FVP of $\mathbf{3 b}$ (recrystallized from MeOH, $195 \mathrm{mg}, 0.60 \mathrm{mmol}, T_{\mathrm{i}} 170{ }^{\circ} \mathrm{C}, T_{\mathrm{f}}$ $600{ }^{\circ} \mathrm{C}, P 0.03 \mathrm{Torr}, t 45 \mathrm{~min}$ ) gave 1 ba; yield: $0.129 \mathrm{mg}(95 \%)$; offwhite solid; mp $195^{\circ} \mathrm{C}$.

${ }^{1} \mathrm{H}$ NMR (DMSO- $d_{6}$ ): $\delta=11.76(\mathrm{~s}, 1 \mathrm{H}$ ), 8.31 (br m, $3 \mathrm{H}$ ), 7.57 (app t, $\left.{ }^{3} J=7.8 \mathrm{~Hz}, 2 \mathrm{H}\right), 7.32\left(\mathrm{~d},{ }^{3} \mathrm{~J}=7.3 \mathrm{~Hz}, 1 \mathrm{H}\right), 6.66(\mathrm{br} \mathrm{s}, 1 \mathrm{H}), 2.68(\mathrm{~s}, 3 \mathrm{H})$.

${ }^{13} \mathrm{C}$ NMR (DMSO- $\left.d_{6}\right): \delta=160.6\left(\mathrm{C}_{\mathrm{q}}\right), 153.1\left(\mathrm{C}_{\mathrm{q}}\right), 150.9(\mathrm{CH}), 142.0\left(\mathrm{C}_{\mathrm{q}}\right)$, $139.5\left(\mathrm{C}_{\mathrm{q}}\right), 128.9(2 \mathrm{CH}), 124.9(\mathrm{CH}), 119.7(2 \mathrm{CH}), 107.4\left(\mathrm{C}_{\mathrm{q}}\right), 103.2$ (CH), $14.3\left(\mathrm{CH}_{3}\right)$.

MS: $m / z(\%)=226\left(\mathrm{M}^{+}, 30\right), 225$ (100), 79 (15), 78 (39).

HRMS: $m / z$ calcd for $\mathrm{C}_{13} \mathrm{H}_{11} \mathrm{~N}_{3} \mathrm{O}\left(\mathrm{M}^{+}\right)$: 225.0897; found: 225.0896 .

1-tert-Butyl-1,7-dihydropyrazolo[3,4-b]pyridin-4-one (1ca)

FVP of 3c (recrystallized from cyclohexane, $300 \mathrm{mg}, 1.02 \mathrm{mmol}, T_{\mathrm{i}}$ $210{ }^{\circ} \mathrm{C}, T_{\mathrm{f}} 500{ }^{\circ} \mathrm{C}, P 0.03 \mathrm{Torr}, t 0.5 \mathrm{~h}$ ) gave 1ca; yield: $185 \mathrm{mg}(95 \%) ;$ yellow solid; $\mathrm{mp} 189-191{ }^{\circ} \mathrm{C}$. 
${ }^{1} \mathrm{H}$ NMR (DMSO- $\left.d_{6}\right): \delta=11.40(\mathrm{~s}, 1 \mathrm{H}), 8.12(\mathrm{br} \mathrm{s}, 1 \mathrm{H}), 8.02(\mathrm{~s}, 1 \mathrm{H})$, 6.49 (br s, $1 \mathrm{H}$ ), $1.74(\mathrm{~s}, 9 \mathrm{H}) \cdot{ }^{13} \mathrm{C}$ NMR (DMSO- $\left.d_{6}\right): \delta=159.3\left(\mathrm{C}_{\mathrm{q}}\right), 152.9$ $\left(\mathrm{C}_{\mathrm{q}}\right), 149.7(\mathrm{CH}), 128.5(\mathrm{CH}), 108.9\left(\mathrm{C}_{\mathrm{q}}\right), 102.2(\mathrm{CH}), 59.6\left(\mathrm{C}_{\mathrm{q}}\right), 29.2(3$ $\mathrm{CH}_{3}$ ).

MS: $m / z(\%)=191\left(\mathrm{M}^{+}, 24\right), 135(56)$.

HRMS: $m / z$ calcd for $\mathrm{C}_{10} \mathrm{H}_{13} \mathrm{~N}_{3} \mathrm{O}\left(\mathrm{M}^{+}\right)$: 191.1064 ; found: 191.1060 .

\section{1,7-Dihydropyrazolo[3,4-b]pyridin-4-one (1cb)}

FVP of 3c (recrystallized from cyclohexane, $50 \mathrm{mg}, 0.17 \mathrm{mmol}, T_{\mathrm{i}}$ $210{ }^{\circ} \mathrm{C}, T_{\mathrm{f}} 750^{\circ} \mathrm{C}, P 0.03$ Torr, $t 0.5 \mathrm{~h}$ ) was followed by distillation of $\mathrm{CH}_{2} \mathrm{Cl}_{2}$ into the U-tube trap. The solvent was removed in vacuo to afford a yellow solid, which was triturated with $\mathrm{Et}_{2} \mathrm{O}$ and filtered under vacuum. The filtrate was further washed with $\mathrm{Et}_{2} \mathrm{O}$ to yield $\mathbf{1} \mathbf{c b}$; yield: $24 \mathrm{mg}$ (67\%); pale brown solid; $\mathrm{mp} 327-330{ }^{\circ} \mathrm{C}$ (Lit. ${ }^{2 \mathrm{~d}} \mathrm{mp} 328-$ $\left.330^{\circ} \mathrm{C}\right)$.

${ }^{1} \mathrm{H}$ NMR $\left(\right.$ DMSO- $\left.d_{6}\right): \delta$ (major tautomer) $=13.54(\mathrm{~s}, 1 \mathrm{H}), 11.71(\mathrm{~s}, 1$ H), 8.28 (br s, $1 \mathrm{H}$ ), 7.62 (br s, $1 \mathrm{H}$ ), 5.66 (br s, $1 \mathrm{H}$ ).

${ }^{13} \mathrm{C}$ NMR (DMSO- $\left.d_{6}\right): \delta$ (major tautomer) $=177.1\left(\mathrm{C}_{\mathrm{q}}\right), 151.2\left(\mathrm{C}_{\mathrm{q}}\right)$, 139.2 (CH), $125.8(\mathrm{CH}), 113.4\left(\mathrm{C}_{\mathrm{q}}\right), 107.3(\mathrm{CH})$.

MS: $m / z(\%)=135\left(\mathrm{M}^{+}, 100\right)$.

\section{1-tert-Butyl-1,7-dihydro-3-methylpyrazolo[3,4-b]pyridin-4-one (1da)}

FVP of 3d (125 mg, $0.41 \mathrm{mmol}, T_{\mathrm{i}} 160{ }^{\circ} \mathrm{C}, T_{\mathrm{f}} 500{ }^{\circ} \mathrm{C}, P 0.03 \mathrm{Torr}, t 1 \mathrm{~h}$ ) gave 1da; yield: $0.70 \mathrm{~g}$ (83\%); off-white solid; $\mathrm{mp} 158^{\circ} \mathrm{C}$.

${ }^{1} \mathrm{H}$ NMR (DMSO- $d_{6}$ ): $\delta=11.17$ (br s, $1 \mathrm{H}$ ), $8.01\left(\mathrm{~d},{ }^{3} \mathrm{~J}=4.7 \mathrm{~Hz}, 1 \mathrm{H}\right.$ ), 6.34 $\left(\mathrm{d},{ }^{3} \mathrm{~J}=4.7 \mathrm{~Hz}, 1 \mathrm{H}\right), 2.45$ (s, $\left.3 \mathrm{H}\right), 1.63(\mathrm{~s}, 9 \mathrm{H})$.

${ }^{13} \mathrm{C}$ NMR (DMSO- $\left.d_{6}\right): \delta=160.7\left(\right.$ br C C $\left._{\mathrm{q}}\right), 152.2\left(\right.$ br C $\left._{\mathrm{q}}\right), 147.5(\mathrm{CH}), 137.4$ (br C $\left.\mathrm{q}_{\mathrm{q}}\right), 107.2\left(\mathrm{br} \mathrm{C}_{\mathrm{q}}\right), 101.5(\mathrm{CH}), 58.5\left(\mathrm{C}_{\mathrm{q}}\right), 28.8\left(3 \mathrm{CH}_{3}\right), 14.3\left(\mathrm{CH}_{3}\right)$.

MS: $m / z(\%)=205\left(\mathrm{M}^{+}, 48\right), 150,(22), 149(100), 148$ (26).

HRMS: $m / z$ calcd for $\mathrm{C}_{11} \mathrm{H}_{15} \mathrm{~N}_{3} \mathrm{O}\left(\mathrm{M}^{+}\right)$: 205.1210; found: 205.1210 .

\section{3-Methyl-1,7-dihydropyrazolo[3,4-b]pyridin-3-one (1db)}

FVP of 3d $\left(100 \mathrm{mg}, 0.33 \mathrm{mmol}, T_{\mathrm{i}} 160{ }^{\circ} \mathrm{C}, T_{\mathrm{f}} 850{ }^{\circ} \mathrm{C}, P 0.03 \mathrm{Torr}, t 45\right.$ min) gave 1db; yield: $0.40 \mathrm{~g}(82 \%)$; off-white solid; $\mathrm{mp} 254^{\circ} \mathrm{C}$.

${ }^{1} \mathrm{H}$ NMR (DMSO- $\left.d_{6}\right): \delta$ (major tautomer) $=13.11(\mathrm{~s}, 1 \mathrm{H}), 11.51(\mathrm{~s}, 1$ H), $7.50\left(\mathrm{t},{ }^{3} \mathrm{~J}=6.3 \mathrm{~Hz}, 1 \mathrm{H}\right), 5.55\left(\mathrm{~d},{ }^{3} \mathrm{~J}=6.3 \mathrm{~Hz}, 1 \mathrm{H}\right), 2.53(\mathrm{~s}, 3 \mathrm{H})$.

${ }^{13} \mathrm{C}$ NMR (DMSO- $\left.d_{6}, 600 \mathrm{MHz}\right): \delta$ (major tautomer) $=178.1\left(\mathrm{br} \mathrm{C}_{\mathrm{q}}\right.$ ), 151.5 (br C $\left.{ }_{\mathrm{q}}\right), 141.5\left(\right.$ br C $\left._{\mathrm{q}}\right), 138.6(\mathrm{CH}), 110.2\left(\mathrm{C}_{\mathrm{q}}\right), 107.1(\mathrm{CH}), 11.0$ $\left(\mathrm{CH}_{3}\right)$.

MS: $m / z(\%)=149\left(\mathrm{M}^{+}, 80\right), 78(100)$.

HRMS: $m / z$ calcd for $\mathrm{C}_{7} \mathrm{H}_{7} \mathrm{~N}_{3} \mathrm{O}\left(\mathrm{M}^{+}\right)$: 149.0584; found: 149.0583 .

\section{1-tert-Butyl-1,7-dihydro-3-phenylpyrazolo[3,4-b]pyridin-4-one (1ea)}

FVP of $3 \mathrm{e}\left(500 \mathrm{mg}, 1.36 \mathrm{mmol}, T_{\mathrm{i}} 220^{\circ} \mathrm{C}, T_{\mathrm{f}} 500{ }^{\circ} \mathrm{C}, P 0.03 \mathrm{Torr}, t 0.5 \mathrm{~h}\right)$ gave 1ea; yield: $358 \mathrm{mg}(96 \%)$; yellow solid; $\mathrm{mp} 295-298^{\circ} \mathrm{C}$.

${ }^{1} \mathrm{H}$ NMR $\left(\mathrm{CDCl}_{3}\right): \delta=11.51(\mathrm{~s}, 1 \mathrm{H}), 8.23\left(\mathrm{~d},{ }^{3} \mathrm{~J}=5.4 \mathrm{~Hz}, 1 \mathrm{H}\right), 8.00(\mathrm{~d}$, $\left.{ }^{3} J=7.3 \mathrm{~Hz}, 2 \mathrm{H}\right), 7.45\left(\mathrm{t},{ }^{3} \mathrm{~J}=7.3 \mathrm{~Hz}, 2 \mathrm{H}\right), 7.36\left(\mathrm{t},{ }^{3} \mathrm{~J}=7.3 \mathrm{~Hz}, 1 \mathrm{H}\right), 6.60$ $\left(\mathrm{d},{ }^{3} \mathrm{~J}=5.4 \mathrm{~Hz}, 1 \mathrm{H}\right), 1.80(\mathrm{~s}, 9 \mathrm{H})$.

${ }^{13} \mathrm{C}$ NMR $\left(\mathrm{CDCl}_{3}\right): \delta=160.0\left(\mathrm{C}_{\mathrm{q}}\right), 154.1\left(\mathrm{C}_{\mathrm{q}}\right), 149.6(\mathrm{CH}), 140.7\left(\mathrm{C}_{\mathrm{q}}\right)$, $134.1\left(\mathrm{C}_{\mathrm{q}}\right), 129.0(2 \mathrm{CH}), 128.4(2 \mathrm{CH}), 128.0(\mathrm{CH}), 105.8\left(\mathrm{C}_{\mathrm{q}}\right), 102.6$ (CH), $59.9\left(\mathrm{C}_{\mathrm{q}}\right), 29.2\left(3 \mathrm{CH}_{3}\right)$.

MS: $m / z(\%)=267\left(\mathrm{M}^{+}, 33\right), 211(100)$.

HRMS: $m / z$ calcd for $\mathrm{C}_{16} \mathrm{H}_{17} \mathrm{~N}_{3} \mathrm{O}\left(\mathrm{M}^{+}\right)$: 267.1366; found: 267.1367 .

\section{1,7-Dihydro-3-phenylpyrazolo[3,4-b]pyridin-4-one (1eb)}

FVP of $3 \mathbf{e}\left(500 \mathrm{mg}, 1.36 \mathrm{mmol}, T_{\mathrm{i}} 220^{\circ} \mathrm{C}, T_{\mathrm{f}} 750{ }^{\circ} \mathrm{C}, P 0.03 \mathrm{Torr}, t 0.5 \mathrm{~h}\right)$ was followed by distillation of $\mathrm{CH}_{2} \mathrm{Cl}_{2}$ into the U-tube trap. The solution was removed and the insoluble product filtered under vacuum to give 1eb; yield: $275 \mathrm{mg}\left(96 \% \mathrm{mix}^{12}\right)$; off-white solid; $\mathrm{mp} 298^{\circ} \mathrm{C}$.

${ }^{1} \mathrm{H}$ NMR (DMSO- $\left.d_{6}\right): \delta$ (major tautomer) $=13.85(\mathrm{br} \mathrm{s}, 1 \mathrm{H}), 11.74(\mathrm{br}$ s, $1 \mathrm{H}$ ), 8.29 (br s, $1 \mathrm{H}), 8.05$ (br d, ${ }^{3} \mathrm{~J}=4.9 \mathrm{~Hz}, 1 \mathrm{H}$ ), 7.63 (m, $\left.2 \mathrm{H}\right), 7.47$ (m, $2 \mathrm{H}), 5.71$ (br s, $1 \mathrm{H})$.

MS: $m / z(\%)=211\left(\mathrm{M}^{+}, 34\right), 183(100)$.

HRMS: $m / z$ calcd for $\mathrm{C}_{12} \mathrm{H}_{9} \mathrm{~N}_{3} \mathrm{O}\left(\mathrm{M}^{+}\right)$: 211.0751 ; found: 211.0751 .

\section{1,3-Di-tert-butyl-1,7-dihydropyrazolo[3,4-b]pyridin-4-one (1fa)}

FVP of $3 f$ ( $500 \mathrm{mg}, 1.43 \mathrm{mmol}, T_{\mathrm{i}} 200{ }^{\circ} \mathrm{C}, T_{\mathrm{f}} 500{ }^{\circ} \mathrm{C}, P 0.03 \mathrm{Torr}, t 0.5 \mathrm{~h}$ ) was followed by distillation of $\mathrm{CH}_{2} \mathrm{Cl}_{2}$ into the U-tube trap. The solution was removed and solvent removed in vacuo to afford 1fa; yield: $345 \mathrm{mg}$ (97\%); yellow solid; $\mathrm{mp} 264-266{ }^{\circ} \mathrm{C}$.

${ }^{1} \mathrm{H} \mathrm{NMR}\left(\mathrm{CDCl}_{3}\right): \delta=11.39(\mathrm{~s}, 1 \mathrm{H}), 8.13\left(\mathrm{~d},{ }^{3} \mathrm{~J}=4.7 \mathrm{~Hz}, 1 \mathrm{H}\right), 6.50(\mathrm{~d}$, $\left.{ }^{3} J=4.7 \mathrm{~Hz}, 1 \mathrm{H}\right), 1.71(\mathrm{~s}, 9 \mathrm{H}), 1.43(\mathrm{~s}, 9 \mathrm{H})$.

${ }^{13} \mathrm{C}$ NMR $\left(\mathrm{CDCl}_{3}\right): \delta=159.3\left(\mathrm{C}_{\mathrm{q}}\right), 154.4\left(\mathrm{C}_{\mathrm{q}}\right), 148.9(\mathrm{CH}), 139.4\left(\mathrm{C}_{\mathrm{q}}\right)$, $105.7\left(\mathrm{C}_{\mathrm{q}}\right), 101.9(\mathrm{CH}), 59.0\left(\mathrm{C}_{\mathrm{q}}\right), 33.7\left(\mathrm{C}_{\mathrm{q}}\right), 29.1\left(3 \mathrm{CH}_{3}\right), 26.1\left(3 \mathrm{CH}_{3}\right)$.

MS: $m / z(\%)=247\left(\mathrm{M}^{+}, 29\right), 232(54), 232(100)$.

HRMS: $m / z$ calcd for $\mathrm{C}_{14} \mathrm{H}_{21} \mathrm{~N}_{3} \mathrm{O}\left(\mathrm{M}^{+}\right)$: 247.1685; found: 247.1690 .

\section{3-tert-Butyl-1,7-dihydropyrazolo[3,4-b]pyridin-4-one (1fb)}

FVP of $3 \mathbf{f}\left(500 \mathrm{mg}, 1.43 \mathrm{mmol}, T_{\mathrm{i}} 200{ }^{\circ} \mathrm{C}, T_{\mathrm{f}} 750{ }^{\circ} \mathrm{C}, P 0.03 \mathrm{Torr}, t 0.5 \mathrm{~h}\right)$ was followed by distillation of $\mathrm{CH}_{2} \mathrm{Cl}_{2}$ into the U-tube trap. The solution was removed and the insoluble product $\mathbf{1} \mathbf{f b}$ was filtered under vacuum; yield: $374 \mathrm{mg}$ (95\% $\left.\mathrm{mix}^{12}\right)$; off-white solid; mp $265-266^{\circ} \mathrm{C}$.

${ }^{1} \mathrm{H}$ NMR $\left(\right.$ DMSO- $\left.d_{6}\right): \delta$ (major tautomer $)=12.88($ br s, $1 \mathrm{H}), 11.56(\mathrm{br}$ s, $1 \mathrm{H}), 7.59$ (br s, $1 \mathrm{H}), 5.63$ (br s, $1 \mathrm{H}), 1.44$ (s, $9 \mathrm{H}$ ).

MS: $m / z(\%)=191\left(\mathrm{M}^{+}, 28\right), 176(100), 149(27)$.

HRMS: $m / z$ calcd for $\mathrm{C}_{10} \mathrm{H}_{13} \mathrm{~N}_{3} \mathrm{O}\left(\mathrm{M}^{+}\right)$: 191.1059; found: 191.1063 .

1-tert-Butyl-4-chloro-3-phenyl-1H-pyrazolo[3,4-b]pyridine (9) Compound 1ea $(1.00 \mathrm{~g}, 3.74 \mathrm{mmol})$ was dissolved in $\mathrm{POCl}_{3}(18 \mathrm{~mL}$, $197 \mathrm{mmol}$ ) and heated to reflux for $4 \mathrm{~h}$. The solution was cooled, and the volume reduced in vacuo. $\mathrm{H}_{2} \mathrm{O}(40 \mathrm{~mL})$ was added slowly to the dark residue followed by sat. aq $\mathrm{NaHCO}_{3}(40 \mathrm{~mL})$. The aqueous layer was extracted with $\mathrm{CH}_{2} \mathrm{Cl}_{2}(3 \times 30 \mathrm{~mL})$, the combined organic layers were washed with brine $(30 \mathrm{~mL})$, dried $\left(\mathrm{MgSO}_{4}\right)$, and the solvent removed in vacuo to afford 9; yield: $1.06 \mathrm{~g}$ (99\%); brown solid; mp 123$125^{\circ} \mathrm{C}$.

${ }^{1} \mathrm{H} \mathrm{NMR}\left(\mathrm{CDCl}_{3}\right): \delta=8.41\left(\mathrm{~d},{ }^{3} \mathrm{~J}=5.0 \mathrm{~Hz}, 1 \mathrm{H}\right), 7.77(\mathrm{~m}, 2 \mathrm{H}), 7.48(\mathrm{~m}, 3$ $\mathrm{H}), 7.14\left(\mathrm{~d},{ }^{3} \mathrm{~J}=5.0 \mathrm{~Hz}, 1 \mathrm{H}\right), 1.91(\mathrm{~s}, 9 \mathrm{H})$.

${ }^{13} \mathrm{C}$ NMR $\left(\mathrm{CDCl}_{3}\right): \delta=151.9\left(\mathrm{C}_{\mathrm{q}}\right), 147.3(\mathrm{CH}), 141.6\left(\mathrm{C}_{\mathrm{q}}\right), 137.7\left(\mathrm{C}_{\mathrm{q}}\right)$, $133.1\left(\mathrm{C}_{\mathrm{q}}\right), 130.5(2 \mathrm{CH}), 128.2(\mathrm{CH}), 127.9(2 \mathrm{CH}), 117.2(\mathrm{CH}), 113.5$ $\left(\mathrm{C}_{\mathrm{q}}\right), 60.7\left(\mathrm{C}_{\mathrm{q}}\right), 29.2\left(3 \mathrm{CH}_{3}\right)$.

MS: $m / z(\%)=287\left[\mathrm{M}^{+}\left({ }^{37} \mathrm{Cl}\right), 10\right], 285\left[\mathrm{M}^{+}\left({ }^{35} \mathrm{Cl}\right), 31\right], 231$ (32), 229 (100).

HRMS: $\mathrm{m} / z$ calcd for $\mathrm{C}_{16} \mathrm{H}_{16}{ }^{35} \mathrm{ClN}_{3}\left(\mathrm{M}^{+}\right)$: 285.1038; found: 285.1039 .

\section{4-Chloro-3-phenyl-1H-pyrazolo[3,4-b]pyridine (10)}

FVP of 9 (50 mg, $\left.0.18 \mathrm{mmol}, T_{\mathrm{i}} 300{ }^{\circ} \mathrm{C}, T_{\mathrm{f}} 750{ }^{\circ} \mathrm{C}, P 0.033 \mathrm{Torr}, t 0.5 \mathrm{~h}\right)$ was followed by distillation of $\mathrm{CH}_{2} \mathrm{Cl}_{2}$ into the U-tube trap. The solvent was removed in vacuo to afford 10; yield: $35 \mathrm{mg}$ (87\%); pale brown solid; mp $274-277^{\circ} \mathrm{C}$. 
${ }^{1} \mathrm{H}$ NMR $\left(\mathrm{CDCl}_{3}\right): \delta$ (major tautomer) $=12.85(\mathrm{~s}, 1 \mathrm{H}), 8.57\left(\mathrm{~d},{ }^{3} \mathrm{~J}=5.2\right.$ $\mathrm{Hz}, 1 \mathrm{H}), 7.81\left(\mathrm{~d},{ }^{3} J=6.2 \mathrm{~Hz}, 2 \mathrm{H}\right), 7.52(\mathrm{~m}, 3 \mathrm{H}), 7.26\left(\mathrm{~d},{ }^{3} J=5.2 \mathrm{~Hz}, 1\right.$ $\mathrm{H})$.

${ }^{13} \mathrm{C}$ NMR $\left(\mathrm{CDCl}_{3}\right): \delta$ (major tautomer) $=153.7\left(\mathrm{C}_{\mathrm{q}}\right), 149.1(\mathrm{CH}), 145.8$ $\left(\mathrm{C}_{\mathrm{q}}\right), 139.2\left(\mathrm{C}_{\mathrm{q}}\right), 132.4\left(\mathrm{C}_{\mathrm{q}}\right), 130.3(2 \mathrm{CH}), 128.7(\mathrm{CH}), 128.1(2 \mathrm{CH})$, $118.3(\mathrm{CH}), 112.4\left(\mathrm{C}_{\mathrm{q}}\right)$.

MS: $m / z(\%)=231$ [M $\left.\mathrm{M}^{+}\left({ }^{37} \mathrm{Cl}\right), 30\right], 229$ [ $\left.\mathrm{M}^{+}\left({ }^{35} \mathrm{Cl}\right), 100\right], 166$ (50).

HRMS: $m / z$ calcd for $\mathrm{C}_{12} \mathrm{H}_{8}{ }^{35} \mathrm{ClN}_{3}\left(\mathrm{M}^{+}\right)$: 229.0412; found: 229.0414 .

\section{1-tert-Butyl-3-phenyl-4-(pyrrolidin-1-yl)-1H-pyrazolo[3,4-b]pyri- dine (11)}

Pyrrolidine (1.0 mL, $11 \mathrm{mmol}$ ) was added to a solution of 9 (250 mg, $0.877 \mathrm{mmol})$ in 1,2-dimethoxyethane $(15 \mathrm{~mL})$ and the mixture was heated at reflux with stirring for $18 \mathrm{~h}$. The solvent was removed and the residue was partitioned between sat. aq $\mathrm{NaHCO}_{3}(25 \mathrm{~mL})$ and EtOAc $(25 \mathrm{~mL})$. The organic layer was separated and the solvent removed in vacuo to yield an orange residue, which was purified by dry flash chromatography eluting with hexane-EtOAc (20:1). Product containing fractions were combined and solvent removed in vacuo to give $\mathbf{1 1}$ as a yellow gum, which crystallized on standing; yield: $87 \mathrm{mg}$ (37\%); mp $122-123{ }^{\circ} \mathrm{C}$.

${ }^{1} \mathrm{H} \mathrm{NMR}\left(\mathrm{CDCl}_{3}\right): \delta=8.18\left(\mathrm{~d},{ }^{3} J=5.5 \mathrm{~Hz}, 1 \mathrm{H}\right), 7.66\left(\mathrm{~d},{ }^{3} \mathrm{~J}=6.9 \mathrm{~Hz}, 2 \mathrm{H}\right)$, $7.42\left(\mathrm{t},{ }^{3} \mathrm{~J}=7.6 \mathrm{~Hz}, 2 \mathrm{H}\right), 7.35\left(\mathrm{t},{ }^{3} \mathrm{~J}=8.6 \mathrm{~Hz}, 1 \mathrm{H}\right), 6.28\left(\mathrm{~d},{ }^{3} \mathrm{~J}=5.5 \mathrm{~Hz}, 1\right.$ $\mathrm{H}), 3.06\left(\mathrm{t},{ }^{3} \mathrm{~J}=6.5 \mathrm{~Hz}, 4 \mathrm{H}\right), 1.89(\mathrm{~s}, 9 \mathrm{H}), 1.73\left(\mathrm{t},{ }^{3} \mathrm{~J}=6.5 \mathrm{~Hz}, 4 \mathrm{H}\right)$.

${ }^{13} \mathrm{C}$ NMR $\left(\mathrm{CDCl}_{3}\right): \delta=153.3\left(\mathrm{C}_{\mathrm{q}}\right), 151.5\left(\mathrm{C}_{\mathrm{q}}\right), 147.4(\mathrm{CH}), 141.8\left(\mathrm{C}_{\mathrm{q}}\right)$, $136.7\left(\mathrm{C}_{\mathrm{q}}\right), 129.2(2 \mathrm{CH}), 128.4(2 \mathrm{CH}), 127.6(\mathrm{CH}), 106.0\left(\mathrm{C}_{\mathrm{q}}\right), 99.6$ $(\mathrm{CH}), 59.7\left(\mathrm{C}_{\mathrm{q}}\right), 51.5\left(2 \mathrm{CH}_{2}\right), 29.1\left(3 \mathrm{CH}_{3}\right), 25.2\left(2 \mathrm{CH}_{2}\right)$.

MS: $m / z(\%)=320\left(\mathrm{M}^{+}, 54\right), 264(100), 263\left(\mathrm{M}-\mathrm{C}_{3} \mathrm{H}_{6} \mathrm{O}, 38\right)$.

HRMS: $m / z$ calc for $\mathrm{C}_{20} \mathrm{H}_{24} \mathrm{~N}_{4}\left(\mathrm{M}^{+}\right)$: 320.2007; found: 320.2005 .

\section{4-Anilino-1-tert-butyl-3-phenyl-1H-pyrazolo[3,4-b]pyridine (12)}

A solution of $9(285 \mathrm{mg}, 1.00 \mathrm{mmol})$, aniline $(0.1 \mathrm{~mL}, 1.1 \mathrm{mmol})$, $\mathrm{Pd}_{2}(\mathrm{dba})_{3}(18 \mathrm{mg}, 0.02 \mathrm{mmol})$, dppp (16 $\left.\mathrm{mg}, 0.04 \mathrm{mmol}\right)$, and $t-$ BuONa (134 mg, $1.4 \mathrm{mmol}$ ) in toluene $(10 \mathrm{~mL})$ contained in an ovendried flask purged with $\mathrm{N}_{2}$, was heated to $70{ }^{\circ} \mathrm{C}$ for $72 \mathrm{~h}$. The mixture was cooled, taken up in $\mathrm{Et}_{2} \mathrm{O}(10 \mathrm{~mL})$, washed with brine $(3 \times 5 \mathrm{~mL})$, and concentrated in vacuo to give the crude product, which was purified by dry flash chromatography eluting with hexane-EtOAc (20:1). Product containing fractions were combined and solvent removed in vacuo to afford $\mathbf{1 2}$ as a yellow gum, which crystallized on standing; yield: $280 \mathrm{mg}(87 \%) ; \mathrm{mp} 116-118^{\circ} \mathrm{C}$.

${ }^{1} \mathrm{H} \mathrm{NMR}\left(\mathrm{CDCl}_{3}\right): \delta=8.23\left(\mathrm{~d},{ }^{3} \mathrm{~J}=5.5 \mathrm{~Hz}, 1 \mathrm{H}\right), 7.68\left(\mathrm{~d},{ }^{3} J=6.9 \mathrm{~Hz}, 2 \mathrm{H}\right)$, $7.77\left(\mathrm{t},{ }^{3} \mathrm{~J}=7.5 \mathrm{~Hz}, 2 \mathrm{H}\right), 7.55\left(\mathrm{t},{ }^{3} \mathrm{~J}=7.4 \mathrm{~Hz}, 1 \mathrm{H}\right), 7.38\left(\mathrm{dd},{ }^{3} J=8.4,7.4\right.$ $\mathrm{Hz}, 2 \mathrm{H}), 7.17$ (m, $3 \mathrm{H}), 6.72$ (d, ${ }^{3} \mathrm{~J}=5.5 \mathrm{~Hz}, 1 \mathrm{H}$ ), 6.62 (br s, $\left.1 \mathrm{H}\right), 1.91$ (s, $9 \mathrm{H})$.

${ }^{13} \mathrm{C}$ NMR $\left(\mathrm{CDCl}_{3}\right): \delta=152.8\left(\mathrm{C}_{\mathrm{q}}\right), 148.8(\mathrm{CH}), 145.9\left(\mathrm{C}_{\mathrm{q}}\right), 140.5\left(\mathrm{C}_{\mathrm{q}}\right)$, $139.2\left(\mathrm{C}_{\mathrm{q}}\right), 134.9\left(\mathrm{C}_{\mathrm{q}}\right), 129.5(2 \mathrm{CH}), 129.3(2 \mathrm{CH}), 129.1(2 \mathrm{CH}), 128.6$ $(\mathrm{CH}), 124.5(\mathrm{CH}), 122.3(2 \mathrm{CH}), 104.5\left(\mathrm{C}_{\mathrm{q}}\right), 97.7(\mathrm{CH}), 60.0\left(\mathrm{C}_{\mathrm{q}}\right), 29.2(3$ $\left.\mathrm{CH}_{3}\right)$.

MS: $m / z(\%)=342\left(\mathrm{M}^{+}, 42\right), 286(100)$

HRMS: $m / z$ calcd for $\mathrm{C}_{22} \mathrm{H}_{22} \mathrm{~N}_{4}\left(\mathrm{M}^{+}\right)$: 342.1850 ; found: 342.1852 .

\section{4-Anilino-3-phenyl-1H-pyrazolo[3,4-b]pyridine (13)}

FVP of 12 (30 mg, $\left.0.88 \mathrm{mmol}, T_{\mathrm{i}} 235^{\circ} \mathrm{C}, T_{\mathrm{f}} 750{ }^{\circ} \mathrm{C}, P 0.03 \mathrm{Torr}, t 0.5 \mathrm{~h}\right)$ was followed by distillation of $\mathrm{CH}_{2} \mathrm{Cl}_{2}$ into the U-tube trap. The solvent was removed in vacuo to afford 13; yield: $18 \mathrm{mg}$ (72\%); pale yellow solid; mp $216-217^{\circ} \mathrm{C}$.
${ }^{1} \mathrm{H} \mathrm{NMR}\left(\mathrm{CDCl}_{3}\right): \delta$ (major tautomer) $=12.92(\mathrm{br} \mathrm{s}, 1 \mathrm{H}), 8.33\left(\mathrm{~d},{ }^{3} \mathrm{~J}=\right.$ $5.7 \mathrm{~Hz}, 1 \mathrm{H}), 7.81\left(\mathrm{~d},{ }^{3} \mathrm{~J}=7.3 \mathrm{~Hz}, 2 \mathrm{H}\right), 7.58\left(\mathrm{t},{ }^{3} \mathrm{~J}=7.4 \mathrm{~Hz}, 2 \mathrm{H}\right), 7.52(\mathrm{t}$, $\left.{ }^{3} J=7.4 \mathrm{~Hz}, 1 \mathrm{H}\right), 7.41\left(\mathrm{t},{ }^{3} \mathrm{~J}=7.9 \mathrm{~Hz}, 2 \mathrm{H}\right), 7.23(\mathrm{~m}, 3 \mathrm{H}), 6.76\left(\mathrm{~d},{ }^{3} \mathrm{~J}=5.7\right.$ $\mathrm{Hz}, 1 \mathrm{H}), 6.74(\mathrm{~s}, 1 \mathrm{H})$.

${ }^{13} \mathrm{C}$ NMR $\left(\mathrm{CDCl}_{3}\right)$ : $\delta$ (major tautomer) $=154.7\left(\mathrm{C}_{\mathrm{q}}\right), 150.3\left(\mathrm{C}_{\mathrm{q}}\right), 146.8$ $(\mathrm{CH}), 144.5\left(\mathrm{C}_{\mathrm{q}}\right) 138.7\left(\mathrm{C}_{\mathrm{q}}\right), 134.4\left(\mathrm{C}_{\mathrm{q}}\right), 129.6(2 \mathrm{CH}), 129.3(2 \mathrm{CH})$, $129.0(2 \mathrm{CH}), 128.9(\mathrm{CH}), 125.1(\mathrm{CH}), 122.8(2 \mathrm{CH}), 102.8\left(\mathrm{C}_{\mathrm{q}}\right), 98.3$ $(\mathrm{CH})$

MS: $m / z(\%)=286\left(\mathrm{M}^{+}, 100\right), 285(58), 258(58)$.

HRMS: $m / z$ calcd for $\mathrm{C}_{18} \mathrm{H}_{14} \mathrm{~N}_{4}\left(\mathrm{M}^{+}\right)$: 286.1224; found: 286.1218 .

\section{Acknowledgment}

We thank Cancer Research UK (studentships to AN and MM; Grant Ref C21383/A6950) for financial support and Lorna Murray for assistance with NMR data.

\section{References}

(1) Deceased.

(2) (a) Donati, D.; Ferrini, S.; Fusi, S.; Ponticelli, F. Synthesis 2003, 2518. (b) Hickey, D. M. B.; Ife, R. J.; Leach, C. A.; Liddle, J.; Pinto, I. L.; Smith, S. A.; Stanway, S. J. Patent PCT Int. Appl. WO 2002030904, 2002; Chem. Abstr. 2002, 136, 325424 (c) Kania, R. S.; Bender, S. L.; Borchardt, A. J.; Braganza, J. F.; Cripps, S. J.; Hua, Y.; Johnson, M. D.; Johnson, T. O. Jr.; Luu, H. T.; Palmer, C. L.; Reich, S. H.; Tempczyk-Russell, A. M.; Teng, M.; Thomas, C.; Varney, M. D.; Wallace, M. B. Patent PCT Int. Appl. WO 2001002369, 2001; Chem. Abstr. 2001, 134, 100864 (d) Dorn, H.; Ozegowski, R. J. Prakt. Chem. 1982, 324, 557. (e) Reimlinger, H.; Peiren, M. A.; Merenyi, R. Chem. Ber. 1970, 103, 3252. (f) Dorn, H.; Zubek, A. Chem. Ber. 1968, 101, 3265. (g) Checchi, S.; Papini, P.; Ridi, M. Gazz. Chim. Ital. 1956, 86, 631.

(3) CDK2 inhibitors: (a) Misra, R. N.; Rawlins, D. B.; Xiao, H. Y.; Shan, W.; Bursuker, I.; Keller, K. A.; Mulheron, J. G.; Sack, J. S.; Tokarski, J. S.; Kimball, S. D.; Webster, K. R. Bioorg. Med. Chem. Lett. 2003, 13, 1133. (b) Misra, R. N.; Xiao, H.; Rawlins, D. B.; Shan, W.; Keller, K. A.; Mulheron, J. G.; Sack, J. S.; Tokarski, L. S.; Kimball, S. D.; Webster, K. R. Bioorg. Med. Chem. Lett. 2003, 13, 2405. $A_{1}$ adenosine antagonists: (c) Tuccinardi, T.; Schenone, S.; Bondavalli, F.; Brullo, C.; Bruno, O.; Mosti, L.; Zizzari, A. T.; Tintori, C.; Manetti, F.; Ciampi, O.; Trincavelli, M. L.; Martini, C.; Martinelli, A.; Botta, M. ChemMedChem 2008, 3, 898. CDK1 and CDK4 inhibitors: (d) Sielecki, T. M.; Boylan, J. F.; Benfield, P. A.; Trainor, G. L. J. Med. Chem. 2000, 43, 1. GSK3 inhibitors: (e) Witherington, J.; Bordas, V.; Gaiba, A.; Naylor, A.; Rawlings, A. D.; Slingsby, B. P.; Smith, D. G.; Takle, A. K.; Ward, R. W. Bioorg. Med. Chem. Lett. 2003, 13, 3059. CHK1 inhibitors: (f) Matthews, T. P.; Klair, S.; Burns, S.; Boxall, K.; Cherry, M.; Fisher, M.; Westwood, I. M.; Walton, M. I.; McHardy, T.; Cheung, K. M.; Van Montfort, R.; Williams, D.; Aherne, G. W.; Garrett, M. D.; Reader, J.; Collins, I. J. Med. Chem. 2009, 52, 4810.

(4) Fischmann, T. O.; Hurza, A.; Duca, J. S.; Ramanathan, L.; Mayhood, T.; Windsor, W. T.; Le, H. V.; Guzi, T. J.; Dwyer, M. P.; Paruch, K.; Doll, R. J.; Lees, E.; Parry, D.; Seghezzi, W.; Madison, V. Biopolymers 2007, 89, 372.

(5) Clarke, D.; Mares, R. W.; McNab, H. J. Chem. Soc., Perkin Trans. 1 1997, 1799.

(6) Review:Gaber, A. M.; McNab, H. Synthesis 2001, 2059. 
Synthesis

M. Mackay et al.

(7) Iwasawa, Y.; Kato, T.; Kawanishi, N.; Masutani, K.; Kouta, M.; Mita, T.; Nonoshita, K.; Ohkubo, M. Patent PCT Int. Appl. WO 2008026769, 2008; Chem. Abstr. 2008, 148, 331690

(8) Adapted from:Wager, T. Patent PCT Int. Appl. WO 2005000303, 2005; Chem. Abstr. 2005, 142, 114054

(9) (a) Adapted from:Niculescu-Duvaz, D.; Springer, C. J.; Gill, A. L.; Taylor, R. D.; Marais, R. M.; Dijkstra, H.; Gaulon, C.; Menard, D.; Roman Vela, E. Patent PCT Int. Appl. WO 2006043090, 2006; Chem. Abstr. 2006, 144, 412508 (b) Suijkerbuijk, B. M. J. M.; Niculescu-Duvaz, I.; Gaulon, C.; Dijkstra, H. P.; Niculescu-Duvaz, D.; Menard, D.; Zambon, A.; Nourry, A.; Davies, L.; Manne, H. A.;
Friedlos, F.; Ogilvie, L. M.; Hedley, D.; Lopes, F.; Preece, N. P. U.; Moreno-Farre, J.; Raynaud, F. I.; Kirk, R.; Whittaker, S.; Marais, R.; Springer, C. J. J. Med. Chem. 2010, 53, 2741.

(10) McNab, H.; Stobie, I. J. Chem. Soc., Perkin Trans. 1 1982, 1845.

(11) Pérez, J. D.; Yranzo, G. I.; Phagouapé, L. M. Bull. Soc. Chim. Fr. 1986, 129.

(12) For $\mathbf{1 e b}$ and $\mathbf{1} \mathbf{f b}$, the product was contaminated with unreacted starting material as well as the pyrazolo[1,5-a]pyrimidine isomer, indicating competing $N$-tert-butyl deprotection prior to ring formation, and hence cyclization via pathway $\mathbf{4}$ to $\mathbf{5}$ in Scheme 1 rather than $\mathbf{4}$ to $\mathbf{1}$.

(13) Chu, I.; Lynch, B. M. J. Med. Chem. 1975, 18, 161. 